\title{
Analysis of the efficacy of resveratrol treatment in patients with scarred uterus
}

\author{
HUASHU MA and ZONGXU QIAO \\ Department of Gynecology, Xingtai People's Hospital, Hebei Medical University \\ Affiliated Hospital Obstetric, Xingtai, Hebei 054001, P.R. China \\ Received April 17, 2017; Accepted September 1, 2017
}

DOI: $10.3892 /$ etm.2018.6126

\begin{abstract}
Scarred uterus is caused by cesarean section surgery, and this condition affects further gestation and delivery in patients. Previous evidence suggested that resveratrol, a polyphenol compound, presents beneficial outcomes for patients with scarred uterus. Therefore, the aim of the present study was to analyze the efficacy of resveratrol in the treatment of patients with scarred uterus. The efficacy of resveratrol in the formation of new vessels and re-epithelialization of the endometrium was analyzed. The present results demonstrated that resveratrol treatment reduced uterus scarring in the majority of patients $(87.36 \%)$ compared with the control. It was also observed that the plasma levels of $\beta$-human chorionic gonadotropin were downregulated by resveratrol treatment in patients with scarred uterus. Furthermore, resveratrol treatment promoted the remodeling of the scarred uterus, the regeneration of the endometrium and improved pregnancy outcomes. In conclusion, the findings of the current study indicate that resveratrol treatment may be a potential strategy for the treatment of scarred uterus patients, which contributes to the improvement of pregnancy outcomes.
\end{abstract}

\section{Introduction}

Severe injuries of the uterus that lead to scar formation may consequently result in infertility or pregnancy complications $(1,2)$. A scarred uterus is mainly caused by cesarean section, as well as by myomectomy, uterine perforation or hysterotomy following the surgery (3). Pregnancy subsequent to scarring of the uterus may lead to uterine rupture, postpartum hemorrhage and placenta previa (4). It has also been reported that pregnancy in scarred uterus patients may result in injury, infection, adhesion increasing, poor healing of the incision and surgical complications during delivery (5). There

Correspondence to: Dr Zongxu Qiao, Department of Gynecology, Xingtai People's Hospital, Hebei Medical University Affiliated Hospital Obstetric, 16 Hongxing Street, Xingtai, Hebei 054001, P.R. China

E-mail: zongxuqiaohos@aliyun.com

Key words: scarred uterus, resveratrol, endometrium, pregnancy are few effective methods to treat scarred uterus due to the scar hypertrophy $(6,7)$. Therefore, exploring more efficient treatments may contribute to the recovery of scarred uterus patients.

Resveratrol is a naturally existing polyphenol, which has presented therapeutic effects in the treatment of uterine diseases $(8,9)$. Previous studies have also indicated that resveratrol is a multifunctional compound with reported beneficial outcomes in various human diseases $(10,11)$. Numerous benefits of resveratrol have been identified in the treatment of human diseases, including cancer therapy, neurodegenerative diseases, cerebrovascular function, type II diabetes mellitus and gestational diabetes mellitus $(12,13)$. Long-term resveratrol treatment prevents ovariectomy-induced osteopenia in rats without hyperplastic effects on the uterus (14). In addition, Novakovic et al (15) have demonstrated the beneficial effects of resveratrol on the contractility of non-pregnant rat uterus for the contribution of $\mathrm{K}^{+}$channels. Furthermore, Zeng et al (16) have indicated that the resveratrol could reduce collagen in fibroblasts, which led to further inhibition of cell growth, cell cycle arrest and apoptosis, thus suggesting that resveratrol is a potential agent for hypertrophic scar treatment. These previous findings suggest that resveratrol treatment may contribute to the recovery of scarred uterus patients.

In the present study, the therapeutic effects of resveratrol in patients with a scarred uterus were investigated. The efficacy of resveratrol in the formation of new vessels and re-epithelialization of the endometrium was reported. The findings in the present study suggest that resveratrol treatment may assist in the remodeling of the scarred uterus and improve pregnancy outcomes.

\section{Materials and methods}

Ethical approval and participant consent. A total of 78 patients with scarred uterus were recruited into the present clinical analysis. The current study was implemented according to the Guide for the Care and Use of Clinical Patients of China (17). The study was approved by the Ethical Committee of Hebei Medical University Affiliated Hospital Obstetric (approval no. 20150320WS). The study included patients with scarred uterus. However, patients with chronic hypertension, coagulation abnormality, twin pregnancy, active labour and high-risk pregnancy were excluded from the present study. All patients 
were required to provide signed informed consent prior to participation.

Study design. The 78 patients with scarred uterus were randomly divided into two groups, and received treatment with placebo $(n=32)$ or resveratrol $(n=46$; Sigma-Aldrich; Merck KGaA, Darmstadt, Germany; $10 \mathrm{mg} /$ day) during a 3-month study period (between May 2015 and July 2015).

ELISA. Plasma samples were obtained prior to- and 3 months post-treatment. Samples were prepared immediately by centrifugation of peripheral venous blood at 2,000 x $\mathrm{g}$ at $4^{\circ} \mathrm{C}$ for $10 \mathrm{~min}$. The serum levels of tumor necrosis factor $\alpha$ (TNF- $\alpha$; cat. no. GERPN2788; Sigma-Aldrich; Merck KGaA), hemoglobin (cat. no. MAK115, Sigma-Aldrich; Merck KGaA) and $\beta$-human chorionic gonadotropin $(\beta$-hCG; cat. no. RAB0092-1KT; Sigma-Aldrich; Merck KGaA) were analyzed in patients with scarred uterus using ELISA kits according to the manufacturer's protocol. The serum concentration levels of these cytokines were measured by an enzyme microplate reader at $570 \mathrm{~nm}$.

Principles and settings of ultrasound diagnosis. An ultrasound diagnosis system was used to analyze contrast-enhanced ultrasound clinical trials using a preprogrammed setting prior to- and 3 months post-treatment. Contrast agent Optison (GE healthcare; Chicago, IL, USA) was taken before $1 \mathrm{~h}$. The preprogrammed setting was optimized to scan the uterus, and the mechanical index was set at $0.2-0.4 \mathrm{MHz}$ to achieve the optimum efficiency. The details of principles and settings of ultrasound were as described in a previous study (18). An Acuson Sequoia 512 ultrasound system (Siemens, AG, Munich, Germany) was used to generate the images. Contrast-specific harmonic software was used to analyze images (Version 3.0; GE Healthcare).

Statistical analysis. Statistical analysis was performed using SPSS version 19.0 software (IBM Corp., Armonk, NY, USA) and Excel (Microsoft Corporation, Redmond, WA, USA). All data are reported as the mean \pm standard error of the mean. Statistical tests for data analysis included Fisher's exact test, log-rank test, $\chi^{2}$ test and Student's two-tailed t-test. $\mathrm{P}<0.05$ was considered to indicate a difference that was statistically significant.

\section{Results}

Characteristics of patients with scarred uterus. A total of 78 patients with scarred uterus were recruited into the present clinical analysis. The mean age of patients was 30.4 years (range, 22-38.8 years old), and the majority of patients had a history of previous cesarean delivery (62 patients, $79.5 \%$ ). In addition, 48 patients with scarred uterus had a history of abortion. All patients were voluntarily divided into two groups and received treatment with resveratrol $(n=46)$ or placebo $(n=32)$. The characteristics of patients with scarred uterus are summarized in Table I.

Resveratrol treatment efficiently decreases inflammation in patients with scarred uterus. A previous study has indicated
Table I. Characteristics of patients with scarred uterus.

\begin{tabular}{lc}
\hline Parameters & Value (n, \%) \\
\hline Patients, $\mathrm{n}$ & 78 \\
Age, years & $30.4 \pm 8.4$ \\
Cesarean delivery (n) & $62(79.5)$ \\
History of abortion, $\mathrm{n}$ & $48(61.5)$ \\
Treatment, $\mathrm{n}$ & \\
Resveratrol & $46(59.0)$ \\
Placebo & $32(41.0)$ \\
\hline
\end{tabular}

that inflammation serves an important role during uterine injury (19). In the current study, the anti-inflammation efficacy of resveratrol was examined in patients with scarred uterus prior to- and 3 months post-treatment. The hemoglobin levels were significantly decreased in patients received treatment of resveratrol (10 mg/day) compared with the placebo group (Fig. 1A). Furthermore, the plasma TNF- $\alpha$ levels were markedly downregulated in the resveratrol group compared with the placebo group (Fig. 1B). These outcomes suggest that resveratrol treatment may decrease inflammation in patients with scarred uterus.

Resveratrol treatment improves the scarred uterus. The results revealed that the plasma levels of $\beta$-hCG were downregulated by resveratrol treatment in patients with scarred uterus after treatment for 3 months (Fig. 2A). In addition, it was demonstrated that the majority of scarred uterus patients $(87.36 \%)$ that received resveratrol treatment presented improvement of the scarred uterus, as indicated by the significantly reduced uterine wall thickness (Fig. 2B). Patients receiving treatment with resveratrol (10 $\mathrm{mg} /$ day) presented significantly reduced irregular menstruation compared with that prior to treatment, whereas patients in the placebo effect demonstrated no evident alterations in the irregularity of menstruation (Fig. 2C). These outcomes suggest that resveratrol treatment is beneficial for the treatment of scarred uterus.

Resveratrol treatment promotes remodeling of the scarred uterus. The efficacy of resveratrol treatment in patients with scarred uterus was investigated by ultrasound examination after treatment for 3 months. It was observed that resveratrol treatment promoted the remodeling of the scarred uterus (Fig. 3A). The results further revealed that the regeneration of the endometrium, muscular cells and vascularization were improved by the treatment with resveratrol (Fig. 3B-D). These outcomes suggest that resveratrol treatment may promote remodeling of the scarred uterus in clinical patients.

Resveratrol treatment improves pregnancy outcomes. A total 42 patients who wanted to achieve pregnancy subsequent to treatment were analyzed in the present study after treatment for 3 months. Among these, 23 scarred uterus patients received resveratrol treatment and 19 patients received a placebo. It was observed that resveratrol treatment improved the pregnancy rate compared with patients receiving placebo 

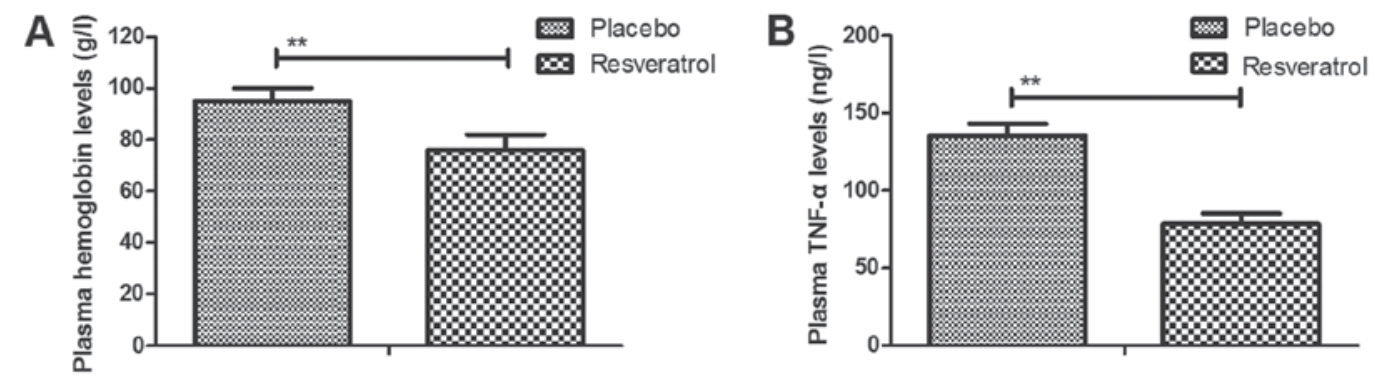

Figure 1. Resveratrol treatment efficiently decreased inflammation in patients with scarred uterus. (A) Hemoglobin levels were decreased in patients receiving treatment with resveratrol (10 mg/day) compared with the placebo group. (B) Resveratrol treatment downregulated the plasma TNF- $\alpha$ levels in patients with scarred uterus. TNF- $\alpha$, tumor necrosis factor $\alpha .{ }^{* * *} \mathrm{P}<0.01$.

A

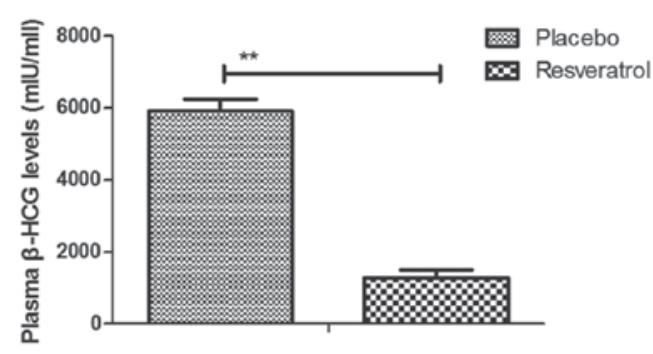

C

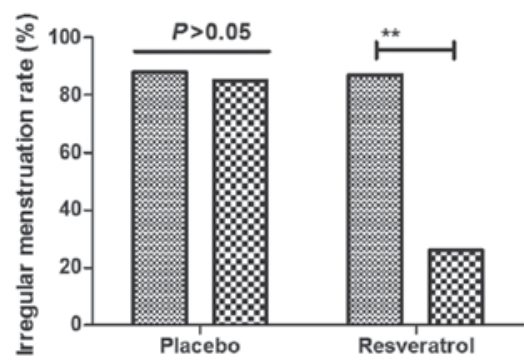

B

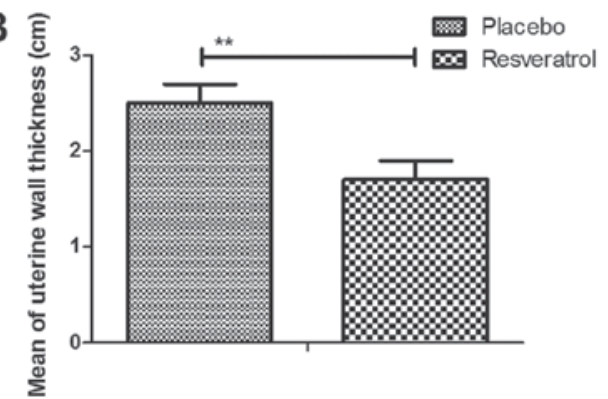

Figure 2. Resveratrol treatment improved the scarred uterus. (A) Resveratrol treatment downregulated the plasma levels of $\beta$-hCG in patients with scarred uterus. (B) Resveratrol treatment decreased the uterine wall thickness. (C) Resveratrol treatment significantly improved the irregular menstruation rate compared with that prior to treatment in patients with scarred uterus. ${ }^{* *} \mathrm{P}<0.05$ vs placebo. $\beta$-hCG, $\beta$-human chorionic gonadotropin.

treatment (Fig. 4A). Vaginal bleeding was markedly reduced and the normal frequency of uterine contractions was markedly reduced in patients who received resveratrol treatment as compared with the patients who received a placebo (Fig. 4B and $\mathrm{C}$ ). These outcomes suggest that resveratrol treatment contributes to the improvement of pregnancy outcomes in patients with scarred uterus.

\section{Discussion}

Earlier studies have indicated that resveratrol presents therapeutic effects in patients with gynecological diseases $(8,9)$. In the present study, the therapeutic effects of resveratrol were further analyzed in patients with a scarred uterus. The current findings demonstrated that resveratrol treatment was able to decrease inflammation in patients with scarred uterus, suggesting that resveratrol treatment was beneficial in the treatment of scarred uterus. Furthermore, a previous study has indicated that contrast-enhanced ultrasound can assist in separately assessing ischemia-induced microvascular remodeling (20). Thus, the current study analyzed the remodeling using contrast-enhanced ultrasound, and the results indicated that resveratrol treatment promoted the remodeling of the scarred uterus and contributed to the improvement of pregnancy outcomes in patients with a scarred uterus. These observations suggest that resveratrol may be an efficient drug for the treatment of scarred uterus patients.

Resveratrol is a multifunctional compound that has been reported to present beneficial outcomes for patients with metabolic and inflammatory diseases. Zhao et al (14) have indicated that long-term resveratrol treatment led to the prevention of ovariectomy-induced osteopenia in rats without hyperplastic effects on the uterus. Another previous study indicated that the long-term (4 months) therapeutic of resveratrol improved patient outcomes (21). In addition, it has been observed that recovery of the scarred uterus can be achieved within 3 months after hysteroscopic resection (22). In the present study, a treatment period of 3 months was selected based on previous results regarding the therapeutic effect of resveratrol. It was revealed that resveratrol treatment significantly improved the scarred uterus and irregular menstruation. Furthermore, Tran et al (23) have suggested that resveratrol is able to ameliorate the chemical and microbial induction of inflammation and insulin resistance in human placenta, adipose tissue and 
A
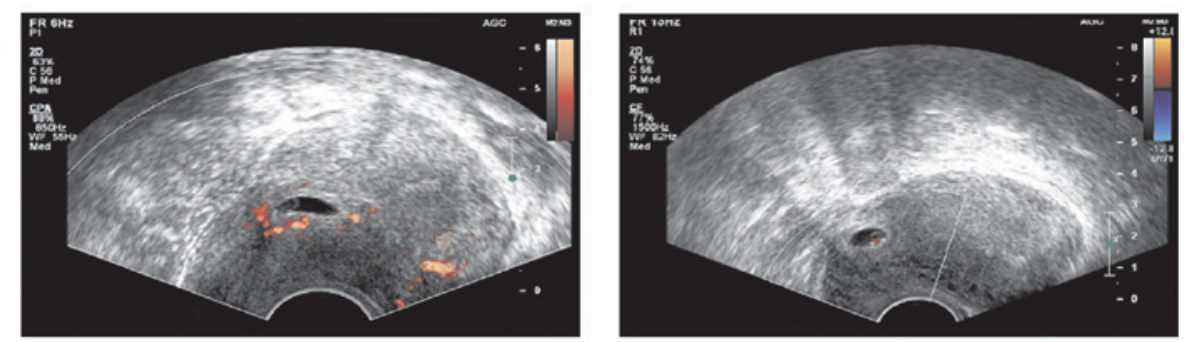

B
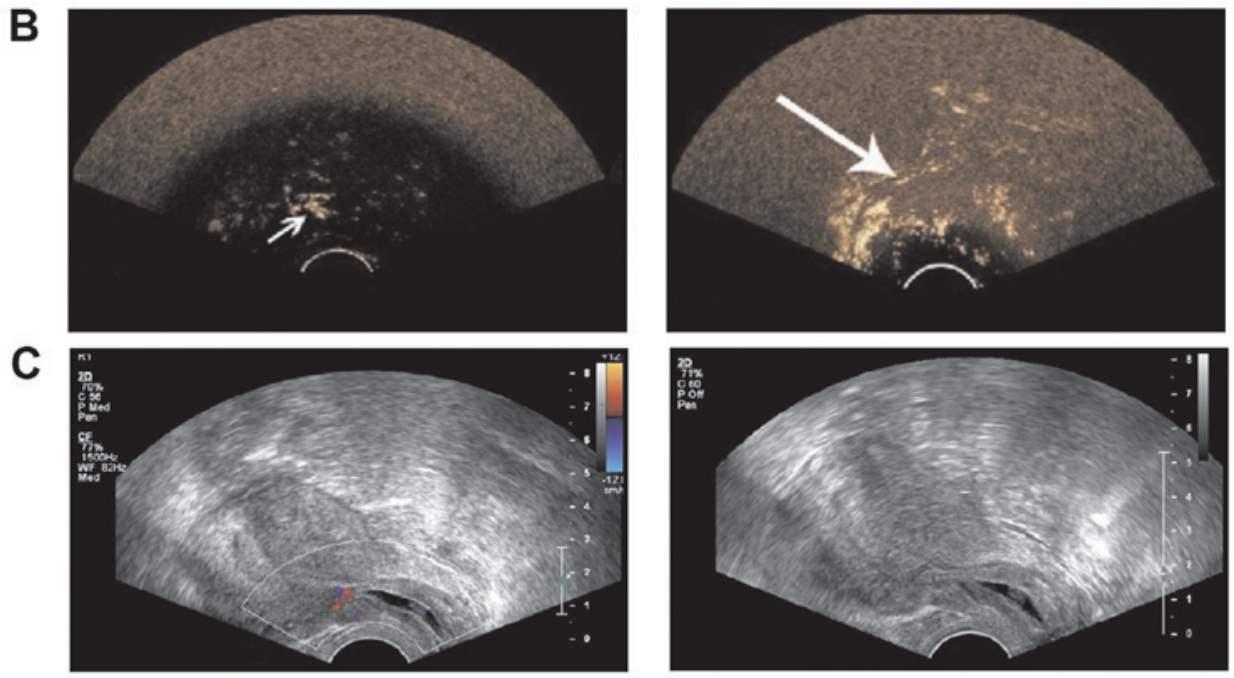

D
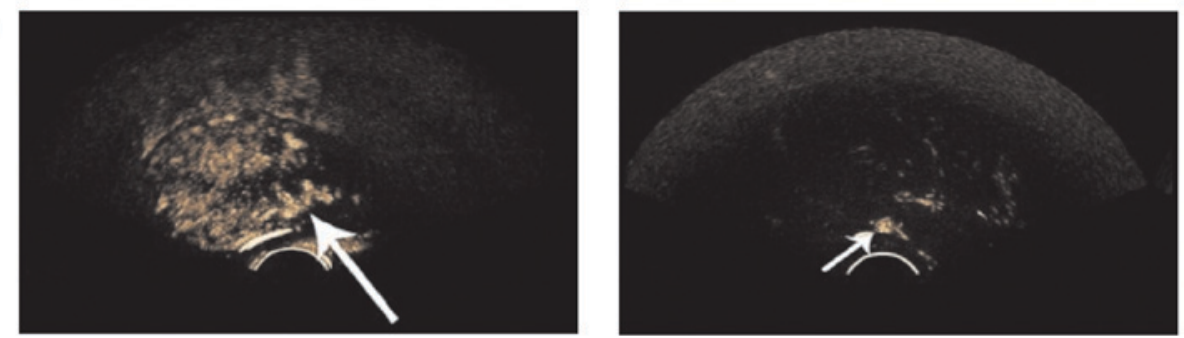

Figure 3. Resveratrol treatment promoted the remodeling of the scarred uterus, as observed by contrast-enhancement ultrasound examination following 3 months of treatment. (A) Resveratrol treatment promoted remodeling of the scarred uterus. Red patches indicated the remodeling of scars. (B) Resveratrol treatment improved the regeneration of the endometrium. (C) Resveratrol treatment improved muscular cells. (D) Resveratrol treatment improved vascularization in patients with a scarred uterus. Arrows indicated the blood vessels in the uterus. Left, placebo group; right, Resveratrol group.
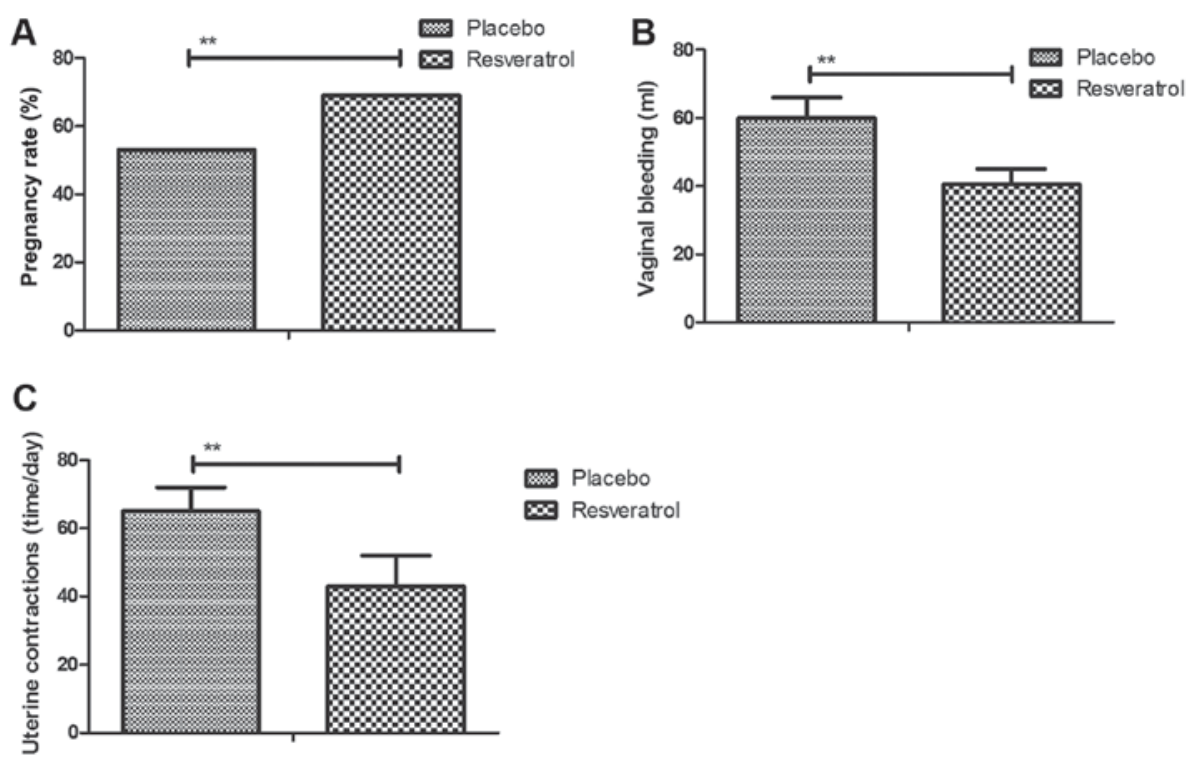

Figure 4. Resveratrol treatment improves the pregnancy outcomes. (A) Resveratrol treatment improved the pregnancy rate compared with patients receiving a placebo. (B) Resveratrol treatment decreased vaginal bleeding in patients with scarred uterus. (C) Resveratrol treatment improved the frequency of uterine contractions in patients with scarred uterus. ${ }^{* *} \mathrm{P}<0.05$ vs placebo. 
skeletal muscle. In the current study, the $\beta$-hCG and TNF- $\alpha$ levels were significantly decreased in patients who received resveratrol treatment (10 $\mathrm{mg} /$ day) compared with the placebo group. However, a limitation of the current study is that the levels of $\beta$-hCG and TNF- $\alpha$ were measured in the patient plasma samples rather than in endometrial fluid samples. Therefore, the levels of these markers may not necessarily correlate with their site-specific expression.

There are currently few effective methods and no specific treatments for scarred uterus patients. Singh et al (24) have indicated that resveratrol treatment modulated the expression of prostaglandin-endoperoxide synthase 2 and the cellular proliferation in the endometrium in a AKT-dependent manner. Resveratrol is a potent inhibitor of vascularization and cell proliferation in experimental endometriosis (25). The results of the current study demonstrated that the regeneration of the endometrium, muscular cells and vascularization were improved by treatment with resveratrol. The findings also suggested that resveratrol treatment improved the pregnancy rate and markedly reduced vaginal bleeding.

In conclusion, the present study demonstrated the beneficial effects of resveratrol in patients with scarred uterus. Resveratrol treatment not only decreased inflammation, but also improved the pregnancy outcomes for patients with scarred uterus. However, further investigation is required to identify the efficacy of resveratrol in a larger cohort of patients with scarred uterus.

\section{References}

1. Wali AS and Naru TY: Silent uterine rupture of scarred uterus-an unusual presentation as amniocele. J Ayub Med Coll Abbottabad 25: 204-205, 2013.

2. Cogan A, Barlow P, Benali N, Murillo D, Manigart Y, Belhomme $\mathrm{J}$ and Rozenberg S: An audit about labour induction, using prostaglandin, in women with a scarred uterus. Arch Gynecol Obstet 286: 1399-1406, 2012.

3. Ofir K, Sheiner E, Levy A, Katz M and Mazor M: Uterine rupture: Differences between a scarred and an unscarred uterus. Am J Obstet Gynecol 191: 425-429, 2004.

4. Skrablin S, Banovic V and Kuvacic I: Successful pregnancy after spontaneous rupture of scarred uterus following fundal myomectomy. Eur J Obstet Gynecol Reprod Biol 121: 251-252, 2005.

5. Grossetti E, Vardon D, Creveuil C, Herlicoviez M and Dreyfus M: Rupture of the scarred uterus. Acta Obstet Gynecol Scand 86 572-578, 2007.

6. Shantha N and Depares J: An unusual case of tear in the pouch of Douglas following spontaneous vaginal delivery in a previously scarred uterus. J Obstet Gynaecol 27: 87-88, 2007.

7. Marinoni E, Santoro M, Vitagliano MP, Patella A, Cosmi EV and Di Iorio R: Intravaginal gemeprost and second-trimester pregnancy termination in the scarred uterus. Int J Gynaecol Obstet 97: 35-39, 2007.

8. Poudel R, Stanley JL, Rueda-Clausen CF, Andersson IJ, Sibley CP, Davidge ST and Baker PN: Effects of resveratrol in pregnancy using murine models with reduced blood supply to the uterus. PLoS One 8: e64401, 2013.

9. Zhao H, Niu Q, Li X, Liu T, Xu Y, Han H, Wang W, Fan N, Tian Q, Zhang $\mathrm{H}$ and Wang Z: Long-term resveratrol consumption protects ovariectomized rats chronically treated with D-galactose from developing memory decline without effects on the uterus. Brain Res 1467: 67-80, 2012.
10. Huang FC, Kuo HC, Huang YH, Yu HR, Li SC and Kuo HC: Anti-inflammatory effect of resveratrol in human coronary arterial endothelial cells via induction of autophagy: Implication for the treatment of Kawasaki disease. BMC Pharmacol Toxicol 18: 3, 2017.

11. Knobloch J, Wahl C, Feldmann M, Jungck D, Strauch J, Stoelben E and Koch A: Resveratrol attenuates the release of inflammatory cytokines from human bronchial smooth muscle cells exposed to lipoteichoic acid in chronic obstructive pulmonary disease. Basic Clin Pharmacol Toxicol 114: 202-209, 2014.

12. Carrizzo A, Puca A, Damato A, Marino M, Franco E, Pompeo F, Traficante A, Civitillo F, Santini L, Trimarco V and Vecchione C: Resveratrol improves vascular function in patients with hypertension and dyslipidemia by modulating NO metabolism. Hypertension 62: 359-366, 2013.

13. Gulvady AA, Ciolino HP, Cabrera RM and Jolly CA: Resveratrol inhibits the deleterious effects of diet-induced obesity on thymic function. J Nutr Biochem 24: 1625-1633, 2013.

14. Zhao H, Li X, Li N, Liu T, Liu J, Li Z, Xiao H and Li J: Long-term resveratrol treatment prevents ovariectomy-induced osteopenia in rats without hyperplastic effects on the uterus. Br J Nutr 111: 836-846, 2014.

15. Novakovic R, Ilic B, Beleslin-Cokic B, Radunovic N, Heinle H, Scepanovic R and Gojkovic-Bukarica L: The effect of resveratrol on contractility of non-pregnant rat uterus: The contribution of $\mathrm{K}(+)$ channels. J Physiol Pharmacol 64: 795-805, 2013.

16. Zeng G, Zhong F, Li J, Luo S and Zhang P: Resveratrol-mediated reduction of collagen by inhibiting proliferation and producing apoptosis in human hypertrophic scar fibroblasts. Biosci Biotechnol Biochem 77: 2389-2396, 2013.

17. Fan X, Li X, Wan H, Hu D, Zhang YP, Volpe AR and DeVizio W: Clinical investigation of the anticaries efficacy of a $1.14 \%$ sodium monofluorophosphate (SMFP) calcium carbonate-based dentifrice: A two-year caries clinical trial on children in China. J Clin Dent 19: 134-137, 2008

18. Keator CS, Lindner JR, Belcik JT, Bishop CV and Slayden OD: Contrast-enhanced ultrasound reveals real-time spatial changes in vascular perfusion during early implantation in the macaque uterus. Fertil Steril 95: 1316-1321.e1-3, 2011.

19. Sahebkar A and Emami SA: Medicinal plants for the treatment of uterus inflammation: Implications from Iranian folk medicine. J Acupunct Meridian Stud 6: 1, 2013.

20. Pascotto M, Leong-Poi H, Kaufmann B, Allrogen A, Charalampidis D, Kerut EK, Kaul S and Lindner JR: Assessment of ischemia-induced microvascular remodeling using contrastenhanced ultrasound vascular anatomic mapping. J Am Soc Echocardiogr 20: 1100-1108, 2007.

21. Dyck JR and Schrauwen P: Resveratrol: Challenges in translating pre-clinical findings to improved patient outcomes. Biochim Biophys Acta 1852: 1069-1070, 2015.

22. Vervoort AJ, Van der Voet LF, Witmer M, Thurkow AL, Radder CM, van Kesteren PJ, Quartero HW, Kuchenbecker WK, Bongers MY, Geomini PM, et al: The HysNiche trial: Hysteroscopic resection of uterine caesarean scar defect (niche) in patients with abnormal bleeding, a randomised controlled trial. BMC Womens Health 15: 103, 2015.

23. Tran HT, Liong S, Lim R, Barker G and Lappas M: Resveratrol ameliorates the chemical and microbial induction of inflammation and insulin resistance in human placenta, adipose tissue and skeletal muscle. PLoS One 12: e0173373,2017.

24. Singh M, Parent S, Leblanc V and Asselin E: Resveratrol modulates the expression of PTGS2 and cellular proliferation in the normal rat endometrium in an AKT-dependent manner. Biol Reprod 84: 1045-1052, 2011.

25. Rudzitis-Auth J, Menger MD and Laschke MW: Resveratrol is a potent inhibitor of vascularization and cell proliferation in experimental endometriosis. Hum Reprod 28: 1339-1347, 2013. 